\section{Unstructured Adaptive Meshes: \\ Bad for your Memory?}

Rupak Biswas

Huiyu Feng

Rob Van der Wijngaart

NASA Ames Research Center

ADAPT 03, OCt 11-12 RPI, Troy, NY

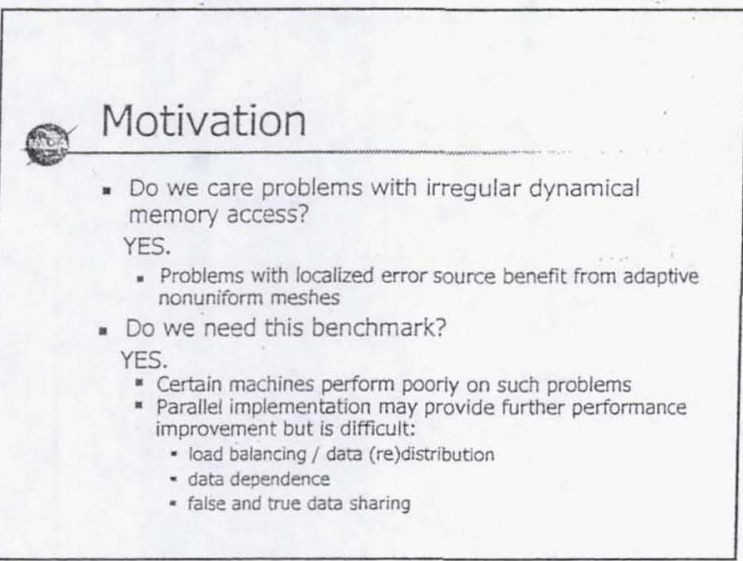

\section{Heat Transfer Problem}

- Mathematical model

$$
\frac{\partial T}{\partial t}+V \cdot \bar{\nabla} T=\varepsilon \nabla^{2} T+S(\mathrm{x}, t)
$$

- Time splitting

$$
\begin{aligned}
& \text { Convection } \frac{\hat{T}-T^{n}}{\Delta t}=-\mathrm{V} \cdot \vec{\nabla} T^{n}+S\left(\mathrm{x}, t^{n}\right) \text { 4th order } R-K \\
& \text { Diffusion } \\
& \frac{T^{n+1}-\hat{T}}{\Delta t}=\varepsilon \nabla^{2} T^{n+1} \\
& \text { Euler implicit }
\end{aligned}
$$

\begin{tabular}{|c|c|c|c|c|c|c|c|c|c|}
\hline benchmark & & & kern & & & & $0 \mathrm{Oax}$ & & new benchmark \\
\hline problems & $\overline{C G}$ & TEP & 15 & MG & FT & LU & SP & BT & $U_{A}$ \\
\hline memory irreqular & 2 & & 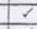 & & & & & & 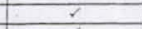 \\
\hline access dynamic & & & 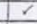 & & & & & & $\checkmark$ \\
\hline scientific aoo. & 5 & & & 18 & 4 & 18 & 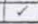 & 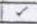 & 1 \\
\hline
\end{tabular}

\section{Background}

- NAS Parallel Benchmarks (NPB, 1991) http://www.nas.nasa.gov/Software/NPB

- Lack in the area of irregular and dynamically changing memory access

\section{Application Selection}

- Representative of problem ciass relevant to scientific computing community

- Simple without sacrificing credibility and effectiveness - Stylized heat transfer problem

- Can be load balanced for range of processor sets with little communication and remapping

- Spectral Element Method (Patera)

- Have irregular, dynamic memory accesses feature. - Adaptive Nonconforming Mesh

\section{Heat Source Term}

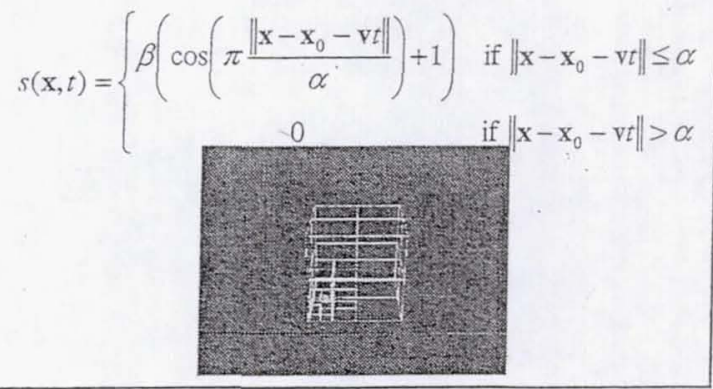



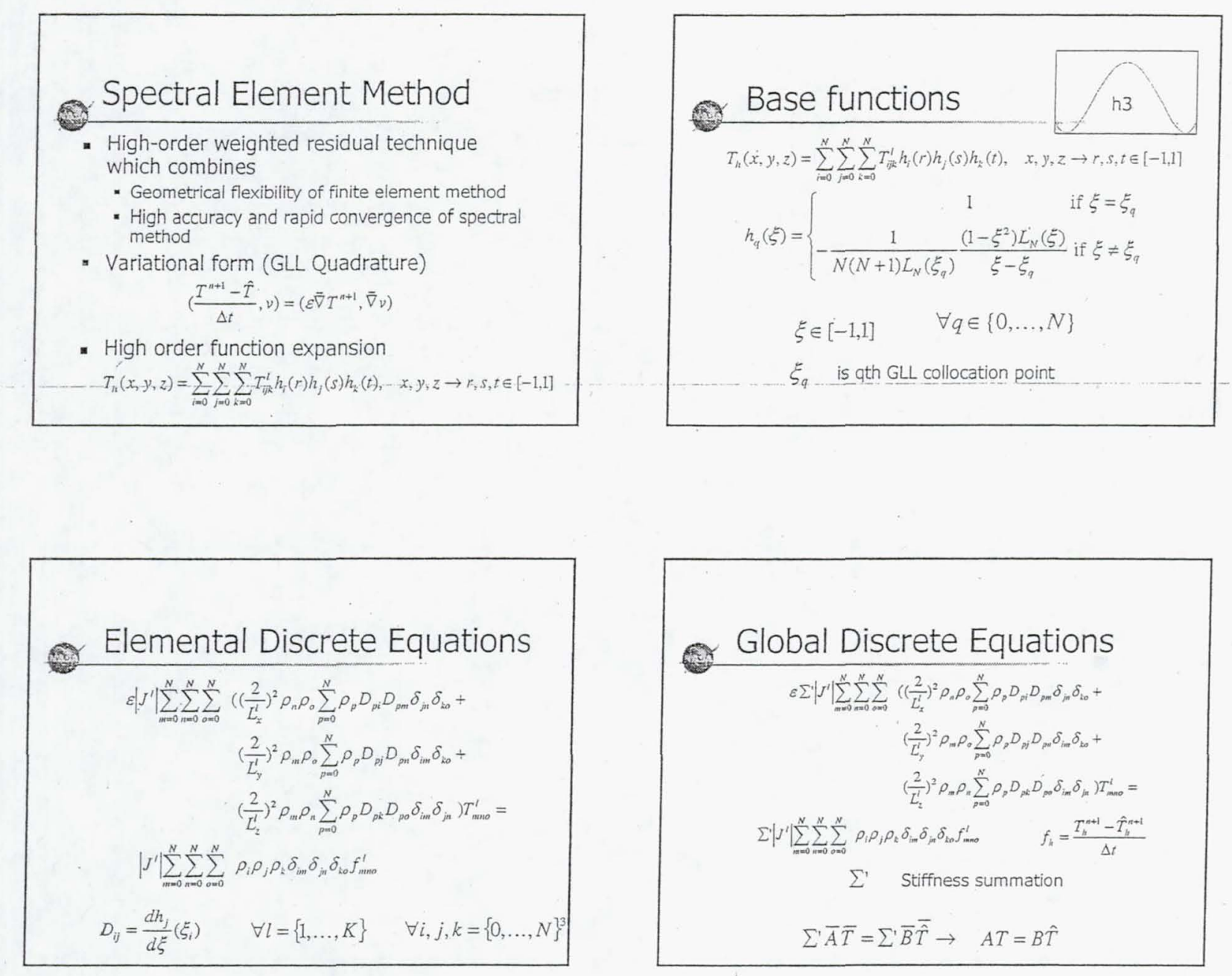

\section{Nonconforming mesh}

- Why nonconforming: local area refinement

- What is nonconforming
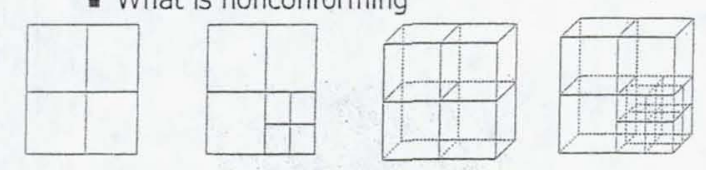

- Problem raised by nonconforming mesh: Continuity across element boundary 

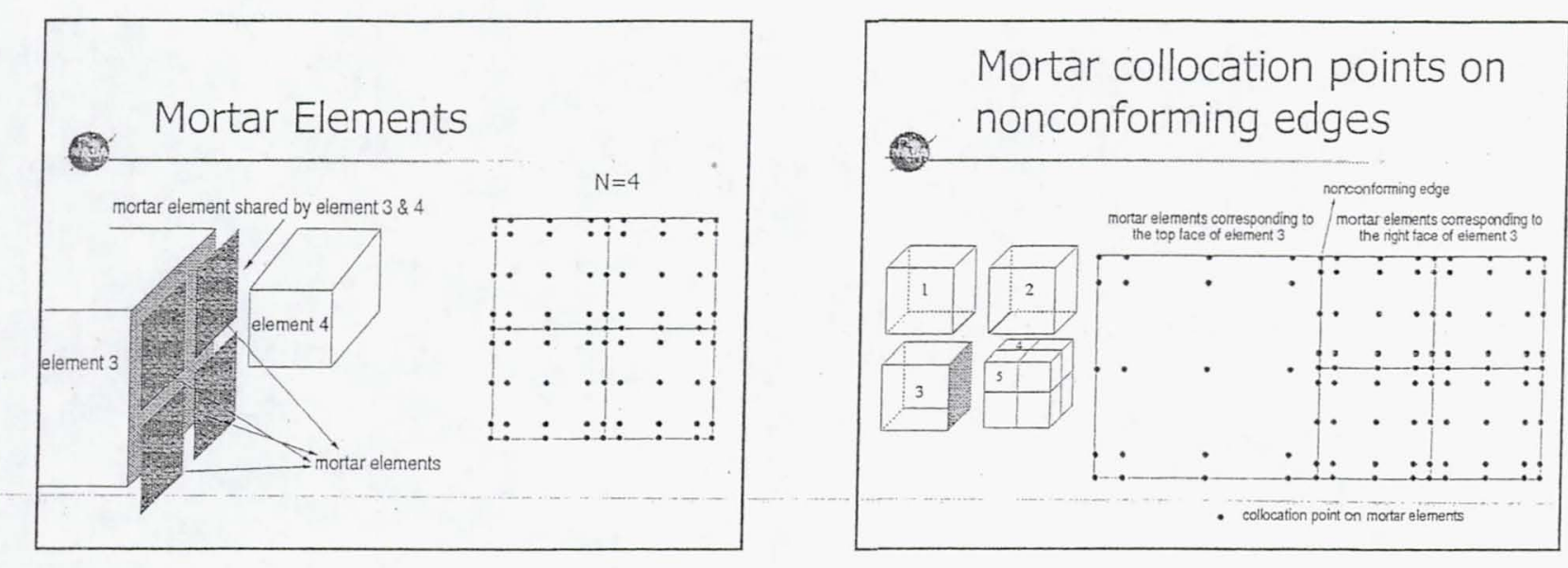

\section{.6. Mortar Element Method}

- Solution on elements

$$
T_{h}(x, y, z)=\sum_{i=0}^{N} \sum_{j=0}^{N} \sum_{k=0}^{N} T_{i j}^{\prime} h_{i}(r) h_{j}(s) h_{k}(t), \quad x, y, z \rightarrow r, s, t \in[-1,1]
$$

- Solution on mortars

$\phi(x, y, z)=\sum_{m=0}^{N} \sum_{n=0}^{N} \phi_{m m}^{p} h_{m}(\hat{r}) h_{n}(\hat{s}), \quad x, y, z \rightarrow \hat{r}, \hat{s} \in[-1,1]$

Mapping for Nonconforming

\section{Faces}

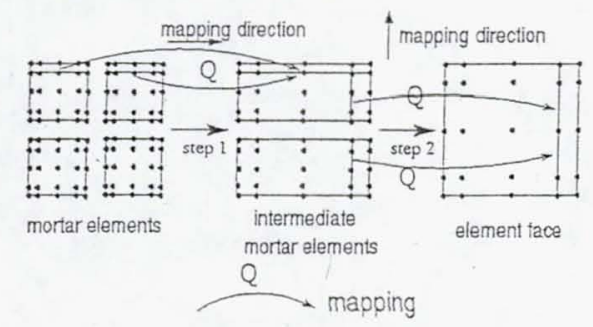

$Q^{\top} \quad$ For reverse mapping from element to mortar
Continuity across nonconforming element iterfaces

$\mathrm{C}^{0}$ continuity is replaced by two conditions:

1. Vertex condition: the solution on an element vertex equals to the solution at the corresponding mortar point.

2. $L^{2}$ condition: the solution difference between an element face and its related mortar elements is minimized in an integral sense.

$$
\begin{aligned}
& \int_{\Gamma^{k},}\left(\left.T\right|_{\Omega^{k}}-\phi\right) \psi d s=0 \\
& \forall l=1, \ldots, 4 \quad \forall \psi \in P_{N-2}\left(\Gamma^{k, J}\right)
\end{aligned}
$$

\section{Discrete Equations}

conforming

nonconforming
Where $\theta$ refers to Global transformation matrix assembled using local transformation matrix $Q$

- symmetrical

- positive definite

Solved by CG with a Diagonal Preconditioner 


\section{Mesh Adaptation Procedure}

- Perform adaptation every $m$ time steps

- Refine elements close to high error region: elements have overlap with the heat source

a Coarsen the grid elsewhere if possible

\section{Adaptation in 3-D (h-type)}
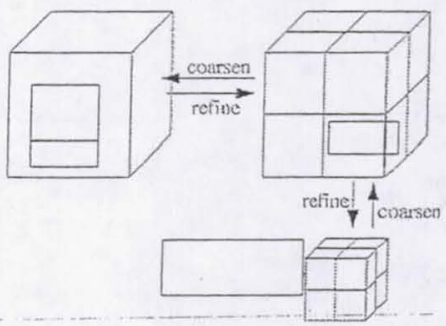

\section{Mesh Adaptation Restrictions}

- The maximal refinement levels/the minimal element size

- Neighboring elements can not differ by more than one level of adaptation.

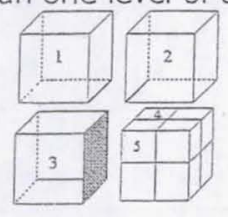

\section{Sample problem}

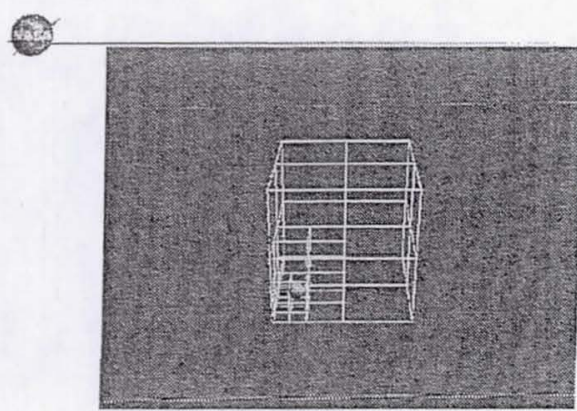

\section{Initial \& Boundary condition}

i Initial grid $[0,1]^{3}$

- Initial temperature $T=0$

- Initial heat source location $(0.30,0.28,0.28)$

- Heat source strength $\beta=10$

- Heat source movement / Velocity field

$$
\mathbf{v}=(1,1,1)
$$

* Boundary condition: T=0@ all faces 

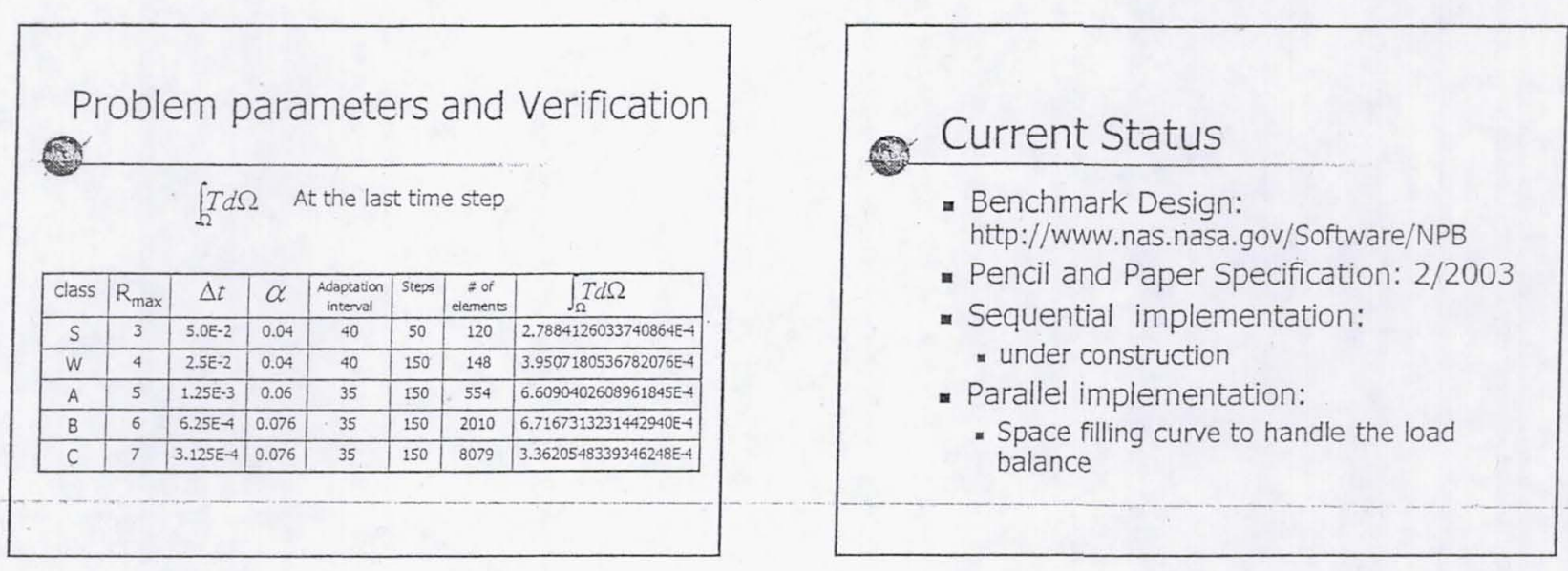

\section{- Acknowledgments}

- This work has been supported by NASA Grant NAG 2-1456, Cooperative Agreement NCC 2-1323.

- Partial support from GWU Center for the Study of Combustion and the Environment. 